\title{
Deep sequencing of tRNA's 3'-termini sheds light on CCA-tail integrity and maturation
}

\author{
ANDREAS CZECH \\ Institute of Biochemistry and Molecular Biology, Chemistry Department, University of Hamburg, 20146 Hamburg, Germany
}

\begin{abstract}
The 3 '-termini of tRNA are the point of amino acid linkage and thus crucial for their function in delivering amino acids to the ribosome and other enzymes. Therefore, to provide tRNA functionality, cells have to ensure the integrity of the $3^{\prime}$-terminal CCA-tail, which is generated during maturation by the $3^{\prime}$-trailer processing machinery and maintained by the CCA-adding enzyme. We developed a new tRNA sequencing method that is specifically tailored to assess the 3'-termini of E. coli tRNA. Intriguingly, we found a significant fraction of tRNAs with damaged CCA-tails under exponential growth conditions and, surprisingly, this fraction decreased upon transition into stationary phase. Interestingly, tRNAs bearing guanine as a discriminator base are generally unaffected by CCA-tail damage. In addition, we showed tRNA species-specific $3^{\prime}$-trailer processing patterns and reproduced in vitro findings on preferences of the maturation enzyme RNase T in vivo.
\end{abstract}

Keywords: tRNA integrity; tRNA maturation; CCA-tail; deep sequencing; CCA-adding enzyme; RNase T

\section{INTRODUCTION}

Transfer RNAs (tRNAs) deliver amino acids for a variety of cellular processes like cell wall synthesis (Dare and lbba 2012), biosynthesis of antibiotics, for example, valanimycin (Garg et al. 2008), and for the transfer of leucine or phenylalanine to the amino terminus of proteins as degradation signal by the L/F transferase (Varshavsky 2011; Dougan et al. 2012). However, the most important function of tRNAs is to transport amino acids to the ribosome for protein synthesis. During ribosomal protein synthesis, tRNAs specifically recognize codons of mRNA and deliver the cognate amino acid to the growing nascent chain. Thus, tRNAs connect the nucleic acid code with the amino acid world. Specifically, this connection lies at the 3 '-end of tRNAs, which bears the invariant CCA-tail in all kingdoms of life. The terminal adenosine nucleotide is coupled with a cognate amino acid by aminoacyl-tRNA synthetases, which form an ester bond between the two (Green and Noller 2002). This bond relies on an intact CCA-tail. Therefore, generating this CCA-tail in tRNA de novo synthesis, as well as maintaining its integrity, is crucial for all tRNA functions.

tRNAs are transcribed as precursor molecules bearing a $5^{\prime}$-leader and $3^{\prime}$-trailer. While the $5^{\prime}$-leader is cleaved in one single enzymatic reaction by the ribonucleoprotein ribonuclease P (Walker and Engelke 2006), processing of

Corresponding author: andreas.czech@chemie.uni-hamburg.de Article is online at http://www.rnajournal.org/cgi/doi/10.1261/rna. 072330.119. the 3 -trailer requires multiple steps being accomplished by multiple enzymes. In bacteria, initially, the endonuclease RNase E cleaves within tRNA 3'-trailers, a processing step which is especially important for multimeric transcripts or long trailer sequences (Li and Deutscher 2002). Subsequently, different exonucleases including RNases $\mathrm{BN}, \mathrm{D}, \mathrm{T}, \mathrm{PH}, \mathrm{II}$, and PNPase, trim the $3^{\prime}$-trailer to its mature length (Reuven and Deutscher 1993a). These exonucleases share redundant activities, which is exemplified by survival of mutant strains with only two out of these six nucleases (Kelly and Deutscher 1992; Reuven and Deutscher 1993a,b). However, besides taking over the function of a deleted enzyme in a knockout strain, certain RNases show specific substrate preferences: While RNase II and PNPase are most efficient on long 3'-trailers (3-7 nt), RNases $\mathrm{T}$ and $\mathrm{PH}$ are the main processors of short trailers (1-4 nt) (Li and Deutscher 1994). Deleting the latter two dramatically reduces the amount of mature tRNA by about $90 \%(\mathrm{Li}$ and Deutscher 1994). Interestingly, these enzymes use different cleavage mechanisms: hydrolytic and phosphorolytic, respectively, and while RNase $T$ can process $+1,+3$, and +4 tRNA precursors, RNase $\mathrm{PH}$ efficiently acts on +2 precursors (Li and Deutscher 1994). Although much knowledge on sequence specificity - especially of RNase T-has

(C) 2020 Czech This article is distributed exclusively by the RNA Society for the first 12 months after the full-issue publication date (see http:// rnajournal.cshlp.org/site/misc/terms.xhtml). After 12 months, it is available under a Creative Commons License (Attribution-NonCommercial 4.0 International), as described at http://creativecommons.org/licenses/by-nc/4.0/. 
been accumulated (Deutscher et al. 1985; Kelly and Deutscher 1992; Zuo and Deutscher 2002; Duh et al. 2015; Wellner et al. 2018), so far we do not know whether certain tRNA precursors prefer maturation by a specific RNase, and whether this might play a role in the regulation of mature tRNAs.

In many organisms, the invariant CCA-tail is not encoded in tRNA genes and has to be added by the crucial tRNA nucleotidyl transferase (CCA-adding enzyme) after 3'-trailer processing. In Escherichia coli, however, all tRNAs are encoded with CCA-tail and thus maturation is ideally completed after 3 '-trailer processing. Hence, the CCA-adding enzyme in E. coli is believed to play no role in the de novo synthesis of tRNAs and is rather important for maintaining the mature tRNA pool by repairing tRNAs with damaged CCA-tails. Such damage is mainly attributed to the tRNA end-turnover carried out by RNase T (Deutscher et al. $1985)$ or by spontaneous autocatalytic cleavage by intramolecular transesterification leading to a $2^{\prime}, 3^{\prime}$-cyclic phosphate group at the $3^{\prime}$-terminus (Lizano et al. 2008). To repair such 3'p-tRNAs, the E. coli CCA-adding enzyme, in contrast to the human homolog, bears a HD domain allowing the removal of the 2',3'-cyclic phosphate group (Aravind and Koonin 1998; Yakunin et al. 2004). The tRNA end-turnover is a quality control mechanism: after removal of the $3^{\prime}$-terminal A by RNase T, the CCA-adding enzyme scrutinizes the tRNA during repair. Only intact, properly modified and folded tRNAs are efficiently repaired. CCA-addition on nicked tRNAs is slower by a factor of 1000 (Dupasquier et al. 2008). These nicked tRNAs remain damaged, are not aminoacylated, not bound by EF-Tu, and not used in translation. In addition, misfolded or hypomodified tRNAs can be tagged by the CCA-adding enzyme with a second CCA entity (Wilusz et al. 2011; Kuhn et al. 2015; Wellner et al. 2018). The resulting CCACCA-tail renders tRNAs susceptible for degradation by RNase $R$ (Wellner et al. 2018).

In spite of the crucial function of tRNA and its central impact on speed and fidelity of protein synthesis (Kirchner et al. 2017), quantification of tRNA lags behind that of other globally important biomolecules like proteins or mRNA. Since tRNAs are very similar in shape, size and charge it is hard to separate them, a prerequisite for specific quantification (Ferro and Ignatova 2015). First, 2D gel electrophoresis (Dong et al. 1996) and microarrays (Dittmar et al. 2004) delivered global quantitative measurements of tRNA. An advantage of the latter technique is, that it also allows assessment of aminoacylation levels (Zaborske et al. 2009; Zaborske and Pan 2010) or integrity of the CCA-tail (Czech et al. 2013). However, a deep sequencing-based quantification is difficult, because the stable secondary structure and the high degree of posttranscriptional modifications, hamper reverse transcription, a crucial step in library preparation (Wilusz 2015). Reports of several attempts to circumvent these obstacles have been published, for example, the usage of demethylation enzyme AlkB to eliminate some hard-stop methylations (ARM-seq) (Cozen et al. 2015) in combination with a highly processive reverse transcriptase (DM-tRNA-seq) (Zheng et al. 2015) or tRNA fractionation to cope with stable secondary structure (HydroSeq) (Gogakos et al. 2017). However, besides methylation, many more modifications remain and might bias tRNA quantification by sequencing.

In this study, we used a new deep sequencing approach to quantify tRNAs and assess their $3^{\prime}$ termini. By sequencing only the hypomodified 3 '-terminal portion of tRNAs from E. coli we avoid biases due to secondary structure and posttranscriptional modifications. Harnessing the potential of this method in assessing the 3'-part of tRNAs, we shed light on CCA-tail integrity and $3^{\prime}$-processing of tRNAs.

\section{RESULTS}

\section{Sequencing $3^{\prime}$-ends of tRNA to reduce sequencing bias}

Sequencing of tRNA is hampered by extensive secondary structure and posttranscriptional modifications (Gogakos et al. 2017). In order to overcome these shortcomings, we developed a new deep sequencing strategy, which analyzes only the 3'-part of tRNAs (Fig. 1A). As applied in $\mathrm{Hy}$ dro-tRNA-seq, tRNAs are fragmented to break secondary structure (Gogakos et al. 2017). However, by ligating the 3 -adapter before fragmentation, we selected only the hypomodified 3'-portion of tRNAs (Supplemental Fig. 1A) for library preparation thereby minimizing biases due to secondary structure and posttranscriptional modifications. For E. coli, mapping the last $10 \mathrm{nt}$ excluding the CCA-tail is sufficient to distinguish between all tRNA species except four isodecoder pairs, Leu ${ }^{C A G}, \mathrm{fMet}^{\mathrm{CAU}}$, Thr ${ }^{\mathrm{CGU}}$, and $\mathrm{Tyr}^{\mathrm{GUA}}$ (Supplemental Fig. 1B). To gain insights into the maturation process and integrity of tRNAs, we used a CCA-adding enzyme knockout strain ( $\Delta \mathrm{cca}$ ) and a ribonuclease T (RNase T) knockout strain ( $\Delta \mathrm{rnt}$ ) in addition to the wildtype CA244 cells and analyzed PAGE-purified tRNA from cells in the exponential (exp) and the stationary (stat) growth phase in two to three replicates with high reproducibility (Supplemental Figs. 2, 3). The abundance of individual tRNA species ranged over two orders of magnitude from very low abundant tRNAs, for example, Arg ${ }^{C C U}$, Gly $^{\mathrm{CCC}}$, or Thr ${ }^{\mathrm{CGU}}$ to high abundant species, for example, $A s{ }^{G U U}$, Asp ${ }^{\mathrm{GUC}}$, or Glu ${ }^{\mathrm{UUC}}$ (Fig. 1B). This is in line with absolute tRNA concentrations reported by Dong et al. (1996). However, the overall correlation between tRNA fractions calculated from this data on the basis of 2D gel electrophoresis and our sequencing data, is rather low $\left(R^{2}=0.07\right)$. To exclude the possibility that this variation is caused by different E. coli strains (CA244 vs. W1485) or media (LB medium vs. defined MOPS medium with supplements) (Dong et al. 
A

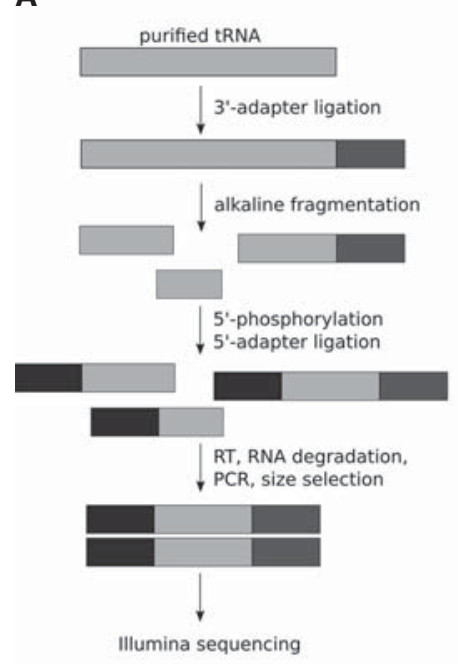

B

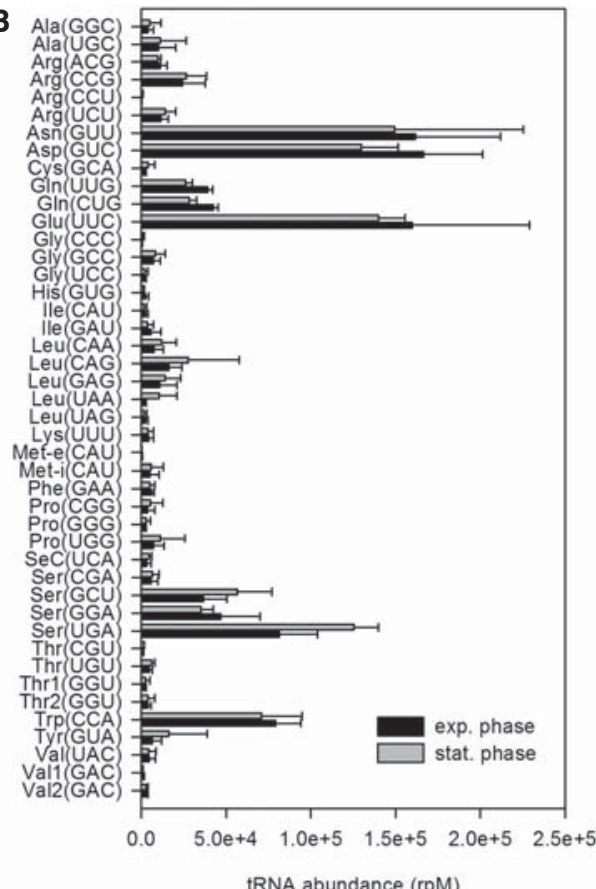

RNA abundance (rpM)

FIGURE 1. Sequencing 3'-portions of tRNA avoids biases derived from secondary structure and posttranscriptional modifications. (A) Scheme of library preparation for deep sequencing. (B) tRNA sequencing reads from wt cells in an exponential and stationary growth phase. Data is represented as means $\pm \mathrm{SD}(n=3)$.

1996), we used 2D gel electrophoresis on our samples to quantify five exemplary tRNAs, Leu ${ }^{\text {CAA }}$, Leu ${ }^{\text {CAG }}$, Leu ${ }^{\text {UAG }}$, Ser ${ }^{G C U}$, and Ser ${ }^{\text {GA }}$ (Supplemental Fig. 4A). Strikingly, tRNA fractions calculated from 2D gel electrophoresis correlated very well while sequencing data of this tRNA subset again showed high divergence (Supplemental Fig. 4B). Since 2D gel electrophoresis does not require any sample processing steps like probe hybridization or reverse transcription, it is very robust and unbiased and thus superior over northern blot or qRT-PCR to evaluate tRNA quantification based on deep sequencing. We reasoned that remaining sequencing bias might derive from fragment generation, adapter ligation or PCR efficiency. We realized that the last $10 \mathrm{nt}$ of Leu ${ }^{\mathrm{CAG}}$ which showed very prominent spots in our 2D gel but had rather little reads in the sequencing experiment, have a high fraction of cytidine residues (70\%). We wondered whether over-representation of a certain nucleotide, high GC-content or stable folding might hamper quantitative cDNA library generation. Thus, we tested whether divergence between 2D gel electrophoresis and deep sequencing results correlates with nucleotide content and folding energy of the $10 \mathrm{nt}$ tRNA fragments in conjunction with $3^{\prime}$-adapter but did not observe any correlation (Supplemental Fig. 4C,D). Furthermore, an incomplete deacylation might leave certain tRNAs aminoacylated and thus unavailable for 3 '-adapter ligation. We compared varying half-life times of amino-
acyl-tRNAs (Hentzen et al. 1972) with the divergence between 2D electrophoresis and deep sequencing, but could not detect any correlation (Supplemental Fig. 4D). In summary, even though we could reduce biases caused by secondary structure and posttranscriptional modifications, there are remaining biases most likely derived from $5^{\prime}$-adapter ligation and varying PCR efficiencies (van Dijk et al. 2014; Bartholomäus et al. 2016). While our method cannot provide a means to derive absolute tRNA quantification, it can be utilized as a method to study relative differences between tRNAs, especially related to the $3^{\prime}$-terminus.

\section{tRNA integrity is diminished in exponential growth phase}

To be active, tRNAs need to be aminoacylated which requires an intact 3'-CCA-tail. Previously, we showed in human HeLa cells, that under oxidative stress the ribonuclease angiogenin is activated, cleaving the $3^{\prime}$ terminal adenosine from tRNA's CCA-tail to quickly inactivate the tRNA pool and consequently inhibit translation (Czech et al. 2013). We aimed to measure tRNA integrity in $E$. coli and used our deep sequencing data to quantify CCA- and CC-bearing tRNA in exponential and stationary phase. tRNA integrity in exponentially growing wildtype $E$. coli cells was rather variable, ranging from 72\%-99\% among different tRNA species (Fig. 2A). As expected, the knockout of the repairing CCA-adding enzyme significantly reduced tRNA integrity (Fig. 2A). One tRNA is particularly affected by the CCA-adding enzyme knockout, tRNA ${ }^{\text {Cys }}$, whose integrity was reduced from $82 \%$ to $40 \%$ (Fig. 2A). This finding might be connected with earlier results showing a translation-independent deacylation of tRNA ${ }^{\text {Cys }}$ by the YbaK deacylase (Ruan and Söll 2005; David et al. 2012). This enzyme is supposed to eliminate mischarged cysteine from tRNA ${ }^{\text {Pro, }}$, but also deacylates correctly charged Cys-tRNA ${ }^{\text {Cys }}$ (Ruan and Söll 2005). Reduced tRNA ${ }^{\text {Cys }}$ charging level has been proven by microarrays (Avcilar-Kucukgoze et al. 2016) and might result in higher susceptibility for exonucleolytic cleavage and a strong dependence on CCA-tail repair by the CCA-adding enzyme. Interestingly, global tRNA integrity increased significantly upon transition of the cells from exponential to stationary phase (Fig. 2A). In contrast, on average the fraction of CC-bearing tRNAs decreased significantly by $\sim 50 \%$ (Fig. 2B). This observation must be 

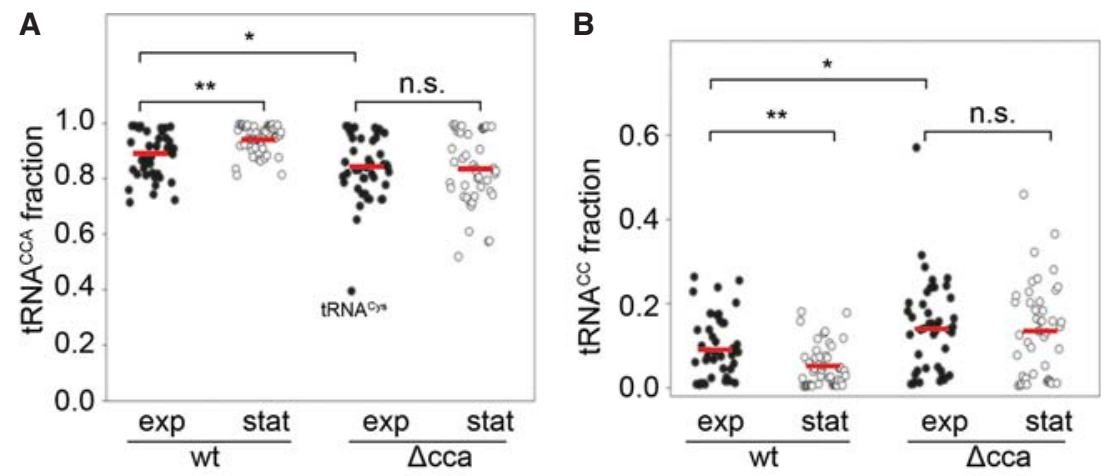

C

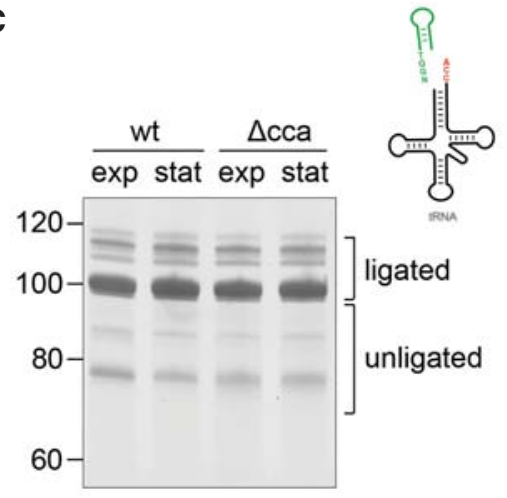

D

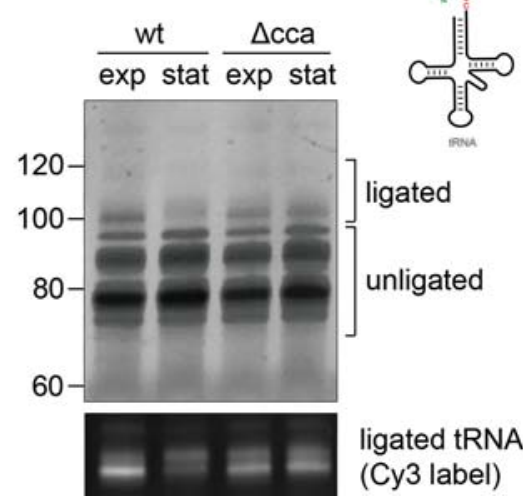

FIGURE 2. CCA-tail integrity varies among tRNA species and increases upon transition from exponential to stationary growth phase. $(A, B)$ Fractions of intact $(A)$ and nonintact $(B)$ tRNAs measured by deep sequencing. Points represent mean fractions of individual tRNAs $(n=3)$, bars represent means among all tRNAs, Wilcoxon rank-sum test. $(C, D)$ Intact $(C)$ and nonintact (D) tRNA identified by ligation to specific oligonucleotides. Ligation was assessed by PAGE, SYBR Gold staining or Cy3 fluorescence detection.

connected with the CCA-adding enzyme, because in $\triangle \mathrm{cca}$ cells, this effect was not visible (Fig. 2A,B). To biochemically verify these results from our deep sequencing data, we adapted the tRNA labeling method developed in the Pan laboratory for tRNA microarrays (Dittmar et al. 2004, 2006), which specifically ligates CCA-containing tRNA to a fluorescent oligonucleotide bearing a 3'-NGGT overhang. First, by reducing the RNA part of the labelling oligonucleotide to only $1 \mathrm{nt}\left(5^{\prime}-\mathrm{rC}\right)$, we dramatically increased the ligation efficiency (Supplemental Fig. 5A). Second, we used oligonucleotides bearing a 3'-NGG overhang, which specifically ligated to nonintact tRNA ${ }^{C C}$, but not to intact tRNA ${ }^{\text {CCA }}$. We proved this method by treating tRNA with angiogenin, which removes the 3 '-terminal adenosine (Czech et al. 2013). While ligation efficiency for the CCA-specific oligonucleotide decreased, the CC-specific oligonucleotide was ligated more efficiently after angiogenin treatment (Supplemental Fig. 5B). We assessed integrity of tRNA, which was used for deep sequencing, in two ligation reactions with oligonucleotides specific for tRNA ${ }^{\mathrm{CCA}}$ and tRNA ${ }^{\mathrm{CC}}$ (Fig. 2C,D). While CCA-specific ligation showed increased ligation products for tRNA of wildtype cells from stationary over exponential growth phase, no difference could be observed for $\Delta$ cca confirming our deep sequencing results (Fig. 2C; Supplemental Fig. 5C). In contrast, CCspecific ligation showed reduced product in wildtype, stationary over exponential phase and no difference for $\Delta$ cca (Fig. 2D; Supplemental Fig. 5C). To evaluate the effect of reduced tRNA integrity on translation efficiency, we applied purified tRNA from exponentially growing or stationary phase cells in an in vitro translation reaction depleted of intrinsic tRNAs and without CCA-adding enzyme (Shimizu et al. 2001). The expression of GFP showed that even slight reduction of CCA integrity in exponential phase tRNA results in reduced protein synthesis (Supplemental Fig. 6). In summary, we verified that a significant fraction of tRNA is not intact under exponential growth conditions leading to reduced protein synthesis and that this fraction is reduced upon transition into stationary phase.

\section{Discriminator base predicts tRNA integrity}

To gain more insights into what governs tRNA integrity, we analyzed our deep sequencing data in more detail and found that the identity of the discriminator base is a good predictor for integrity of tRNAs (Fig. 3). In wildtype cells under exponential growth phase conditions, tRNAs with discriminators adenosine (A), uridine $(U)$, and cytidine (C) showed on average tRNA integrity of only $85 \%$. In contrast, tRNAs with discriminator guanosine (G) showed on average $98.5 \%$ integrity (Fig. 3). These fractions are complemented by tRNA ${ }^{C C}$ fractions, which were high for tRNAs bearing discriminators $A, U$, and $C$ and low for tRNAs bearing discriminator base $G$ (Supplemental Fig. 7). We thought of three possible explanations for this observation: First, varying aminoacylation and consequently EF-Tu binding might result in varying protection of the 3 '-termini from exonuclease cleavage. However, tRNAs from one isoacceptor family, that is, charged by the same aminoacyl-tRNA synthetase, showed different behavior: Of the four tRNA ${ }^{\text {Arg }}$ species, two bear discriminator $G$ and showed high integrity $(99 \%$ for $\mathrm{Arg}^{\mathrm{CCG}}, \mathrm{Arg}^{\mathrm{UCU}}$ ) and two bear discriminator $\mathrm{A}$ and showed low integrity (93\% for $\mathrm{Arg}^{\mathrm{ACG}}, 83 \%$ for $\mathrm{Arg}^{\mathrm{CCU}}$ ). Thus, we concluded that tRNA integrity is not majorly dependent 


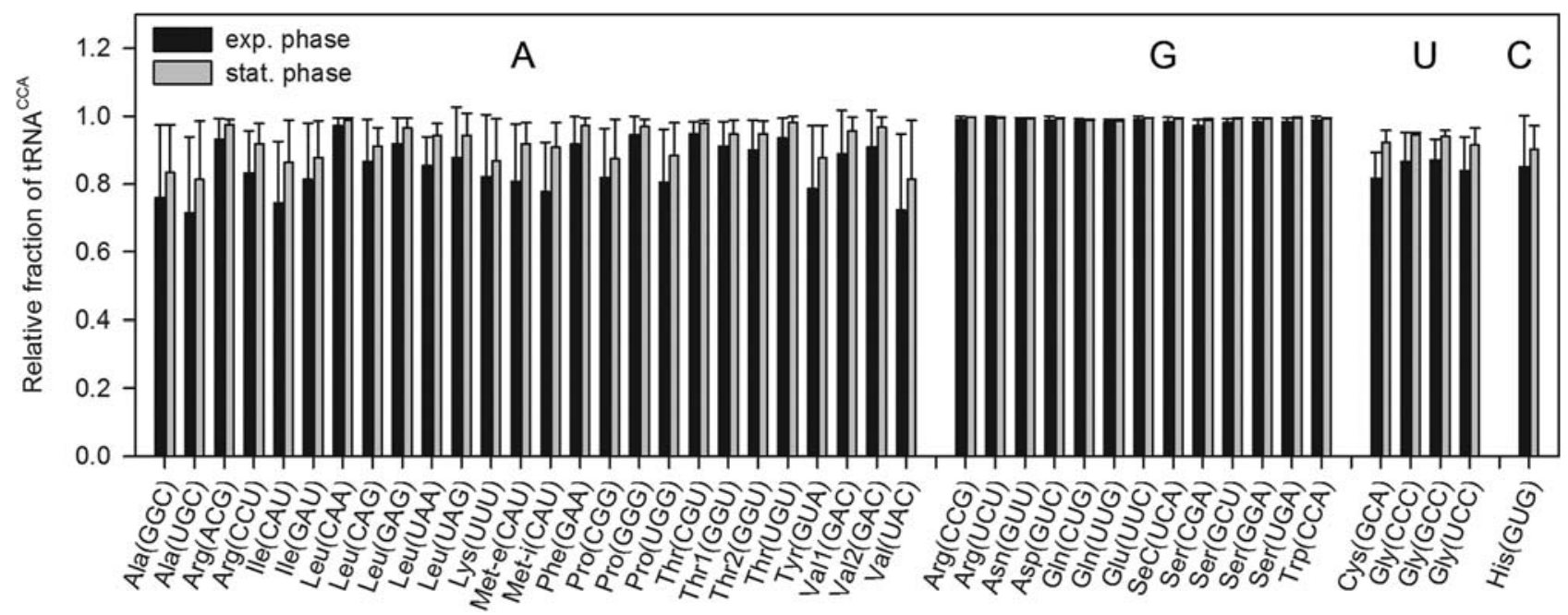

FIGURE 3. The discriminator base predicts CCA-tail integrity. Fractions of intact CCA-tail among different tRNA species measured by deep sequencing. Data is represented as means $\pm \operatorname{SD}(n=3)$.

on the amino acid identity. Second, the CCA-adding enzyme might favor tRNAs with a certain discriminator base. Indeed, it was shown that the human CCA-adding enzyme prefers tRNAs with discriminator A over G (Wende et al. 2015). However, the same study showed equal efficiency of the E. coli CCA-adding enzyme on tRNAs bearing discriminator base $A$ and $G$, eliminating a preference of this enzyme as explanation for our observations (Wende et al. 2015). Third, we reasoned that differences in the acceptor stem stability could lead to unfolding in less stable tRNAs, thus, allowing attack by nucleases. In a previous study on initiator $t R N A^{\text {fMet }}$ it was shown, that mutation of discriminator base from purine to pyrimidine exacerbated stacking onto the acceptor stem, leading to increased breathing of the helix and accessibility by enzymes like Met-tRNA transformylase (Lee et al. 1993). Mutating discriminator base $A$ to $G$ did not show any significant change in stability (Lee et al. 1993), dismissing the effect of the discriminator base on tRNA stability to explain our findings. In addition, we did not measure any significant differences in the overall acceptor stem stability among tRNAs from different discriminator base groups (Supplemental Fig. 8). In summary, it remains elusive what causes variation of $3^{\prime}$ CCA-tail integrity and what is the impact of the discriminator base.

\section{3'-tRNA deep sequencing sheds light on trailer processing}

Since we exclusively sequenced the 3 '-portion of tRNAand due to the high sequencing depth-we obtained many reads from tRNA precursors still containing their 3'trailer. To gain insights into the maturation process of individual tRNAs, we grouped reads according to their trailer length and normalized each group to the amount of ma- ture tRNA. Note that different trailer lengths result in differences in mapping, that is, shorter trailer reads could not be mapped uniquely and were instead assigned to groups of tRNA genes. As expected, the read counts for precursor tRNAs decreased with increasing trailer length reflecting a high processing efficiency (Supplemental Fig. 9A). However, the pattern varied drastically among different tRNA genes. This suggests that processing is not uniform and might be regulated to yield different amounts of mature tRNA. Interestingly, certain tRNA precursors accumulated at a specific trailer length. For example, ThrUGU_43 showed the biggest fraction of very long trailers up to $8 \mathrm{nt}$, which might be caused by a $\mathrm{C}_{7} \mathrm{C}_{8}$-motif which might represent an obstacle for exonucleolytic processing by RNase T (Supplemental Fig. 9A). However, LeuUAA_76 showed an accumulation of 3 nt-trailers, downstream from a $\mathrm{G}_{2} \mathrm{G}_{3}$-motif (Supplemental Fig. 10A). Further research will be necessary to explain accumulation of specific precursor tRNAs. At the first trailer position we found the highest variability among tRNA precursor fractions ranging from $0 \%$ to $17 \%$ of mature tRNAs and intriguingly, all tRNA trailers with very high fractions, for example, ArgACG_64-67, HisGUG_37, and MetCAU_82, started with uridine (Supplemental Fig. 9A). Thus, we analyzed 1-nt-trailer fraction in relation to the identity of this first nucleotide. We found that trailers starting with $U$ are significantly more abundant than all other nucleotides (Fig. $4 A)$, suggesting a slow processing of such precursors in comparison to trailers starting with $A, C$, or $G$. On the other hand, trailers starting with adenosine are most rare and thus might be processed very quickly (Fig. 4A). In vitro experiments by the Deutscher laboratory demonstrated that $\mathrm{RNase} \mathrm{PH}$ and RNase T are the ribonucleases involved in final trimming of precursors (Li and Deutscher 1994). While the substrates of RNase PH are mainly 2 nt- trailers, RNase 

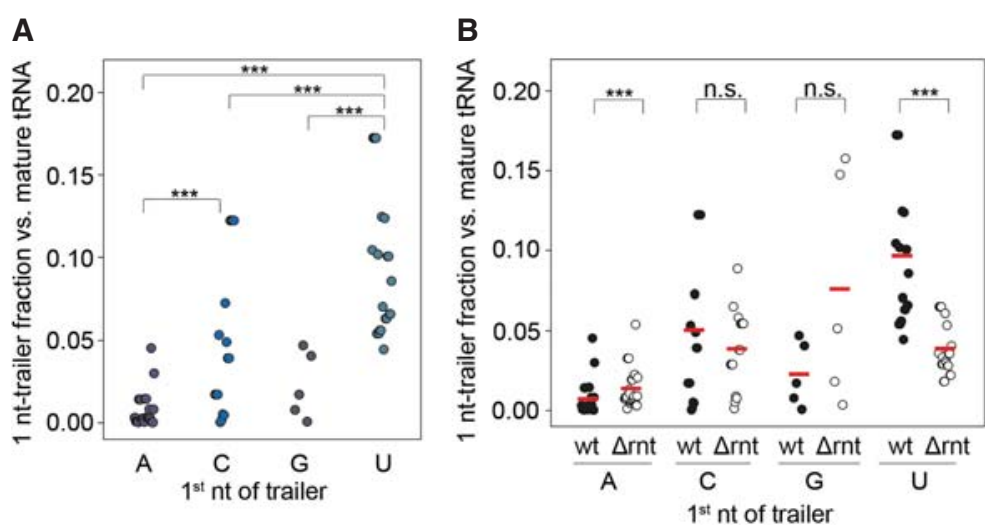

FIGURE 4. 1 nt-trailers are processed by RNase T with preference for adenosine. (A) Fractions of $1 \mathrm{nt}$-trailer precursors grouped by nucleotide identity show preference for adenosine over uridine. Points represent individual $1 \mathrm{nt}$-precursor fractions normalized by respective mature tRNA $(n=2)$, Wilcoxon rank-sum test. (B) Knockout of RNase T significantly increases the fraction of A-starting trailers and decreases U-starting trailers. Points represent individual $1 \mathrm{nt}$-precursor fractions normalized by respective mature tRNA $(n=2)$, bars represent means among all tRNAs within one group, Wilcoxon rank-sum test.

T processes predominantly $1 \mathrm{nt}$-trailers (Li and Deutscher 1994). Our results showed that the latter ribonuclease favors adenosine over uridine at the first trailer position (Fig. 4A), which corroborates in vitro results showing a nearly fivefold higher $k_{c a t} / K_{M}$ of RNase $T$ for $A_{17}$ over $U_{17}$ oligonucleotides (Zuo and Deutscher 2002). To further prove this, we compared fractions of $1 \mathrm{nt}$-trailers of different nucleotides in wildtype and RNase $T$ knockout $(\Delta \mathrm{rnt})$ cells. Indeed, knockout of RNase T significantly increased the fraction of tRNA precursors with one adenosine trailers while uridine trailers were significantly reduced (Fig. 4B). The latter observation might be caused by up-regulation of compensatory ribonucleases which might favor uridine over adenosine. As expected, $1 \mathrm{nt}$-trailers bearing cytidine are not affected by RNase T knockout, since $\mathrm{C}$ and CC motifs block RNase T processing by a factor of 100 and even completely, respectively (Zuo and Deutscher 1999, 2002). Thus, RNase $T$ will not process 1 nt-trailers bearing a cytidine. We wanted to test whether our results are artefacts resulting from varying ligation efficiency of 3 '-adapters to different 3 '-terminal nucleotides, that is, lower ligation efficiency for tRNA precursors ending with $A$ over those ending with $U$. Therefore we repeated our analysis with 2 nt-trailers and hypothesized that a general ligation bias would result in similar results. However, 2 nt-trailer fractions are distributed more equally among the four nucleotides, and no effect or strongly reduced effect of RNase T knockout was observed (Supplemental Fig. 9B). In summary, we showed for the first time varying processing effectivity among different tRNA species in vivo raising the possibility of tRNA pool regulation on the level of maturation. In addition, we reproduced in vitro results in cells showing preferred maturation of A-starting trailers by RNase $T$.

\section{Precursor preference for RNase T might impact CCA-tail integrity}

To connect our findings on tRNA integrity with the results on $3^{\prime}$-maturation, we compared 3 '-trailer sequences of tRNAs with different discriminator bases and observed general similarity among them (Supplemental Fig. 10). However, tRNAs bearing discriminator $G$ have significantly fewer trailers starting with adenosine and more trailers starting with cytidine than tRNAs bearing discriminator A or U (Supplemental Fig. 10). As shown before, RNase T prefers adenosine and disfavors cytidine at the first position of the trailer (Fig. 4B). Thus, precursor tRNAs bearing discriminator $\mathrm{A}$ or $\mathrm{U}$ might be preferentially processed by RNase $T$, while in the case of tRNAs bearing discriminator $\mathrm{G}$, other ribonucleases, most likely RNase $\mathrm{PH}$, might be preferentially involved in $3^{\prime}$-trailer trimming. Subsequently, RNase T might not only process the $3^{\prime}$-trailer, but also cleave the adenosine of the CCA-tail before it is inhibited by the CC-motif, as has been shown in vitro (Li and Deutscher 1994; Zuo and Deutscher 2002). Consequently, tRNAs being processed by RNase T might have a higher risk for CCA-tail damage, which might explain the lower integrity of tRNA bearing discriminator $A$ and $U$, compared to those bearing discriminator $\mathrm{G}$ (Supplemental Fig. 11).

\section{DISCUSSION}

By delivering amino acids for protein synthesis to the ribosome, tRNAs are the central connector between the nucleic acid language of the genetic code and the amino acid language of proteins. Since in ternary complex with EFTu and GTP, tRNAs transport amino acids to ribosomes by diffusion, the speed of incorporation of an amino acid into the growing peptide chain depends mainly on the concentration of cognate tRNA (Anderson 1969; Sørensen et al. 1989). Stretches of slowly translated codons at domain boundaries coordinate cotranslational folding of individual domains and ensure folding fidelity (Zhang et al. 2009). Besides tRNA concentrations, variation in aminoacylation levels (Zaborske et al. 2009) and CCAtail integrity (Czech et al. 2013) can regulate translation under certain environmental conditions such as starvation or oxidative stress (Zaborske et al. 2009; Czech et al. 2013). Quantitative assessment of the tRNA pool by means of deep sequencing is hampered by stable secondary structure and posttranscriptional modifications which influence reverse transcription. Here, we applied a new protocol for 
deep sequencing of tRNA by analyzing only the hypomodified 3'-portion of tRNAs, avoiding biases from secondary structure or modification (Fig. 1; Supplemental Fig. 1A). Unfortunately, our absolute tRNA quantification revealed low correlation with tRNA concentrations determined by 2D gel electrophoresis (Dong et al. 1996). Thus, we have to consider further sources of biases, that is, $5^{\prime}$-adapter ligation and PCR efficiency (Linsen et al. 2009). It was shown that sequencing bias in small RNAs is independent of the sequencing platform, but depends strongly on the library preparation method (Linsen et al. 2009). Although unbiased absolute tRNA quantification was not achieved, we generated highly reproducible, highly enriched reads of $3^{\prime}$-tRNA portions which allowed us to shed light on the integrity of tRNA's CCA-tail and the maturation process of tRNA precursors.

Surprisingly, we found a significant fraction of tRNAs with damaged CCA-tails, that is, CC-tails, in exponentially growing E. coli cells (Fig. 2A,B). We confirmed this result biochemically (Fig. 2C,D) and showed that this tRNA damage impairs protein synthesis in vitro (Supplemental Fig. 6). Intriguingly, only tRNAs bearing discriminator bases $A, U$, or $C$ are affected by CCA-tail damage (Fig. 3; Supplemental Fig. 7) and transition from exponential into stationary growth phase reduces the fraction of damaged tRNA (Figs. 2, 3). This seems counter-intuitive, since in stationary phase translation is shut down by forming inactive hibernating $100 \mathrm{~S}$ ribosomes (Chai et al. 2014; Beckert et al. 2018), and thus a more intact tRNA pool is not needed. However, this shutdown of translation might be what is responsible for the higher tRNA integrity. Since ternary complexes are not used and tRNAs not deacylated by ribosomes during this shutdown, the $3^{\prime}$-termini of tRNAs are constantly protected, preventing cleavage by cellular ribonucleases. Compared with that, under exponential growth conditions, aminoacyl-tRNA synthetases and ribonucleases compete for the free 3 '-terminus of deacylated tRNA leaving the ribosome. Alternatively, shut down of transcription in the stationary phase and degradation of stable RNA reduces the number of tRNA molecules in the cell and might allow repair by the CCA-adding enzyme to be more efficient, thus increasing the fraction of intact tRNAs.

For the first time, we globally assessed tRNAs bearing different lengths of their $3^{\prime}$-trailers from E. coli in vivo. We found high variability among precursor fractions of different tRNA species (Supplemental Fig. 9A) indicating different efficiencies of $3^{\prime}$-trailer processing of different precursors. This might imply maturation of tRNA precursors as the point of regulation of the pool of the mature tRNA. However, we could not explain the accumulation of specific $3^{\prime}$-trailer intermediates. It will be interesting to compare $3^{\prime}$-trailer reads from knockout strains of different processing exonucleases. In addition, in agreement with previous in vitro studies (Li and Deutscher 1994), our precursor data confirmed the major involvement of RNase T in the processing of +1-precursor tRNAs (Fig. 4B) and the preference of this enzyme for adenosine over uridine (Fig. 4A). In addition, different studies showed that exonuclease function of RNase $T$ is inhibited by cytidines (Zuo and Deutscher 2002; Duh et al. 2015; Wellner et al. 2018). In agreement with these findings, we found no significant change in the processing of cytidine trailers upon knockout of RNase T (Fig. 4B), suggesting, that these tRNA precursors are processed by another ribonuclease. Intriguingly, we found an over-representation of adenosine and underrepresentation of cytidine at the beginning of $3^{\prime}$-trailers of tRNAs bearing $A$ and $U$ discriminator bases (Supplemental Fig. 10). Those tRNAs showed CCA-tail damage which might be caused by their preferential maturation by RNase $T$, which might not stop at the CCA-tail but cleaves the terminal adenosine (Deutscher et al. 1985). Such collateral damage during de novo synthesis of tRNAs bearing $A$ and $U$ discriminators must be repaired by the CCA-adding enzyme.

In summary, with our new sequencing protocol we provide a tool to specifically assess the 3 '-termini of tRNAs, which is essential for tRNA functionality. Reduced maturation efficiency of precursors as well as damaged CCA-tails are hard to detect with conventional sequencing methods but have a significant impact on the pool of functional tRNAs (Rak et al. 2018). Environmental stress conditions or antibiotics might affect tRNA's 3'-termini without changing the overall abundances of tRNA species. Hence, our sequencing method provides a tailored tool to detect such effects. In addition, tRNA precursor processing is still widely enigmatic. Thus, studying $3^{\prime}$-trailers in different RNase knockout strains will shed light on the impact of individual processing RNases and the effect of sequence motifs in 3'-trailers on maturation efficiency. This will help to elucidate whether tRNA precursor processing is regulated by cells to adjust the pool of functional tRNAs.

\section{MATERIALS AND METHODS}

\section{Growth conditions and RNA isolation}

E. coli CA244 wt, $\Delta \mathrm{cca}$, and $\Delta \mathrm{rnt}$ cells were grown at $37^{\circ} \mathrm{C}$ in LB medium (10 g/L NaCl, $10 \mathrm{~g} / \mathrm{L}$ tryptone and $5 \mathrm{~g} / \mathrm{L}$ yeast extract). Cells were harvested in exponential $\left(O D_{600} 0.3\right)$ and stationary phase $\left(\mathrm{OD}_{600} 5\right.$ and 3 for wt and $\Delta \mathrm{cca}$, respectively). Total RNA was isolated by hot-phenol extraction or TRIzol (Thermo Fisher Scientific). Total tRNA was purified by cutting respective bands from denaturing polyacrylamide gels and eluted overnight at $4^{\circ}$ $C$ in elution buffer ( $50 \mathrm{mM}$ potassium acetate, $200 \mathrm{mM}$ potassium chloride, $\mathrm{pH}$ 7.0).

\section{tRNA deep sequencing}

Total tRNA was 3'-dephosphorylated with T4 PNK (Thermo Fisher Scientific) for $45 \mathrm{~min}$ at $37^{\circ} \mathrm{C}$ and purified with Clean and 
Concentrator Kit (Zymo Research). A preadenylated sequencing adapter (rApp/TGGAATTCTCGGGTGCCAAGG/3ddC) was ligated to the $3^{\prime}$-end of tRNAs using T4 RNA ligase 2 truncated (NEB) for $2.5 \mathrm{~h}$ at $22^{\circ} \mathrm{C}$. For preparation of the sequencing library which contains $3^{\prime}$-terminal tRNA parts, the sample was subjected to random alkaline fragmentation for $20 \mathrm{~min}$ at $95^{\circ} \mathrm{C}$ in $100 \mathrm{mM}$ $\mathrm{NaHCO}_{3}, 2 \mathrm{mM}$ EDTA pH 9.2. Subsequently, the RNA was 5'-phosphorylated with T4 PNK (NEB) and a second adapter (GUUCAGAGUUCUACAGUCCGACGAUC) was ligated to the $5^{\prime}$-termini with T4 RNA ligase 1 (NEB) at $22^{\circ} \mathrm{C}$ overnight. Using a primer complementary to the $3^{\prime}$-adapter (CCTTGGCACCCGA GAATTCCA) the RNAs were reverse transcribed into cDNA with Revert Aid H Minus RT (Thermo Fisher Scientific) for $1 \mathrm{~h}$ at $44^{\circ} \mathrm{C}$. RNA was degraded by the addition of $\mathrm{NaOH}$ and incubation at $90^{\circ} \mathrm{C}$ for $10 \mathrm{~min}$ and CDNA containing both adapters was amplified by PCR with Pfu DNA polymerase in 10-18 cycles (Thermo Fisher Scientific). Amplicons of 130-140 bp (i.e., 10-20 nt 3'-tRNA part $+120 \mathrm{nt}$ adapters) were gel-purified, analyzed by 2100 Bioanalyzer (Agilent) and Qubit 3.0 (Thermo Fisher Scientific). cDNA libraries were sequenced with TruSeq SBS kits (Illumina) on a HiSeq2000 (Illumina) machine. Sequenced reads were trimmed with fastx-toolkit (0.0.13.2; quality threshold: 20) and sequencing adapters were cut by using cutadapt (1.2.1; minimal overlap: $1 \mathrm{nt})$. tRNA sequences were downloaded from the genomic tRNA database (Escherichia coli K12) and 10 nt 5' upstream of the CCA-tails were used for mapping sequencing reads. For precursor analysis, 9 nt incl. CCA were used with variable lengths of trailer.

\section{tRNA 2D gel electrophoresis and northern blotting}

For the first dimension, $1 \mu \mathrm{g}$ of total RNA was separated fully denatured on $10 \%$ polyacrylamide, $8 \mathrm{M}$ urea in $1 \times$ TAE buffer at $50^{\circ} \mathrm{C}$. Bands of tRNA were excised and layered onto $20 \%$ polyacrylamide, $4 \mathrm{M}$ urea in $1 \times$ TAE buffer. Due to the lower urea concentration tRNAs are allowed to partially refold and run at room temperature for 19-20 h. Gels were stained with SYBR Gold staining (Thermo Fisher Scientific). To identify spots, RNA from 2D gels was transferred onto Amersham Hybond-N membrane (GE Healthcare) in cooled $0.5 \times$ TAE for $2 \mathrm{~h}$ at $80 \mathrm{~V}$ and UV-crosslinked. Membranes were prehybridized for $5 \mathrm{~h}$ in Church buffer $(250 \mathrm{mM}$ $\mathrm{Na}_{2} \mathrm{HPO}_{4} \mathrm{pH} 7.2,1 \mathrm{mM}$ EDTA, 7\% SDS, $0.5[\mathrm{w} / \mathrm{v}] \mathrm{BSA}, 0.08 \mathrm{mg} / \mathrm{mL}$ ssDNA) at $30^{\circ} \mathrm{C}$. Subsequently, Cy3-labeled probes (Leu ${ }^{\mathrm{CAG}}$ : CCCCCACGTCCGTAAGGACACTAACACC, Leu ${ }^{\mathrm{UAG}}$ : CACCTTG CGGCGCCAGAACCTA, Leu ${ }^{\text {CAA: }}$ GGCACGTATTTCTACGGTTG ATTTTGAATCAACTGCGTCTACC, Ser ${ }^{\text {UGA }}$ : CGAACTCTGGAAC

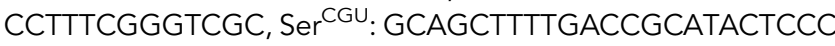
TTAGCAGG) were added to a final concentration of $40 \mathrm{nM}$ and incubated overnight at $30^{\circ} \mathrm{C}$. Membranes were washed twice for $10 \mathrm{~min}$ at $30^{\circ} \mathrm{C}$ in $2 \times \mathrm{SSC}, 0.2 \% \mathrm{SDS}$ and twice for $7 \mathrm{~min}$ at $30^{\circ} \mathrm{C}$ in $1 \times$ SSC, $0.1 \%$ SDS.

\section{tRNA integrity measurement by ligation}

Total RNA was deacylated for $45 \mathrm{~min}$ at $37^{\circ} \mathrm{C}$ in $0.1 \mathrm{M}$ Tris- $\mathrm{HCl} \mathrm{pH}$ 9. For tRNA ${ }^{\mathrm{CC}}$ assessment, RNA was dephosphorylated with T4 PNK (Thermo Fisher Scientific) for $45 \mathrm{~min}$ at $37^{\circ} \mathrm{C}$ and ligated to CC-specific stem-loop oligonucleotides bearing a $3^{\prime}$-GGN overhang with T4 DNA ligase (NEB) for $1 \mathrm{~h}$ at $16^{\circ} \mathrm{C}$. To detect intact
tRNA $^{\text {CCA }}$ we used stem-loop oligonucleotides, which hybridize with their 3'-TGGN overhang to intact CCA-tail. Ligated tRNA was analyzed on denaturing $10 \%$ polyacrylamide gels and visualized by fluorescence (Bio-Rad ChemiDoc MP) and SYBR Gold staining (Thermo Fisher Scientific).

\section{In vitro translation}

To assess activity of isolated tRNA we used the PURExpress $\Delta$ (aa, tRNA) Kit (NEB). Fifteen microliters of reactions including $E$. coli ribosomes, energy, and amino acid mix and GFPencoding plasmid were supplemented with $10 \mu \mathrm{g}$ purified tRNA from exponentially growing and stationary phase cells. The reaction was incubated at $37^{\circ} \mathrm{C}$ for $250 \mathrm{~min}$ in a plate reader and GFP fluorescence was measured simultaneously. Equal amounts of ribosomes and tRNA in each reaction were verified by gel electrophoresis.

\section{DATA DEPOSITION}

RNA-seq data have been deposited at the Gene expression Omnibus under accession number GSE126019.

\section{SUPPLEMENTAL MATERIAL}

Supplemental material is available for this article.

\section{ACKNOWLEDGMENTS}

I thank Karoline Raulf and Alexandra Martitz for excellent technical assistance, Alexander Bartholomäus, Irina Chelysheva, and Johannes Wagner for bioinformatic assistance, and Zoya Ignatova, Mario Mörl, Heike Betat, and Karoline Wellner for scientific discussion and mutant E. coli strains. I thank Peter R. Wills, Robin Thompson, and Robert Rauscher for critical reading of the manuscript. This work was supported by the Deutsche Forschungsgemeinschaft (CZ 234/1-1 to A.C.).

Received June 18, 2019; accepted November 7, 2019.

\section{REFERENCES}

Anderson WF. 1969. The effect of tRNA concentration on the rate of protein synthesis. Proc Natl Acad Sci 62: 566-573. doi:10.1073/ pnas.62.2.566

Aravind L, Koonin EV. 1998. The HD domain defines a new superfamily of metal-dependent phosphohydrolases. Trends Biochem Sci 23: 469-472. doi:10.1016/S0968-0004(98)01293-6

Avcilar-Kucukgoze I, Bartholomäus A, Cordero Varela JA, Kaml RF-X, Neubauer P, Budisa N, Ignatova Z. 2016. Discharging tRNAs: a tug of war between translation and detoxification in Escherichia coli. Nucleic Acids Res 44: 8324-8334. doi:10.1093/nar/gkw697

Bartholomäus A, Del Campo C, Ignatova Z. 2016. Mapping the nonstandardized biases of ribosome profiling. Biol Chem 397: 23-35. doi:10.1515/hsz-2015-0197

Beckert B, Turk M, Czech A, Berninghausen O, Beckmann R, Ignatova Z, Plitzko JM, Wilson DN. 2018. Structure of a hibernating $100 \mathrm{~S}$ ribosome reveals an inactive conformation of the ribosomal 
protein S1. Nat Microbio/ 3: 1115-1121. doi:10.1038/s41564-0180237-0

Chai Q, Singh B, Peisker K, Metzendorf N, Ge X, Dasgupta S, Sanyal S. 2014. Organization of ribosomes and nucleoids in Escherichia coli cells during growth and in quiescence. J Biol Chem 289: 1134211352. doi:10.1074/jbc.M114.557348

Cozen AE, Quartley E, Holmes AD, Hrabeta-Robinson E, Phizicky EM, Lowe TM. 2015. ARM-seq: AlkB-facilitated RNA methylation sequencing reveals a complex landscape of modified tRNA fragments. Nat Methods 12: 879-884. doi:10.1038/nmeth.3508

Czech A, Wende S, Mörl M, Pan T, Ignatova Z. 2013. Reversible and rapid transfer-RNA deactivation as a mechanism of translational repression in stress. PLoS Genet 9: e1003767. doi:10.1371/journal .pgen.1003767

Dare K, Ibba M. 2012. Roles of tRNA in cell wall biosynthesis. Wiley Interdiscip Rev RNA 3: 247-264. doi:10.1002/wrna.1108

David A, Das SR, Gibbs JS, Bennink JR, Yewdell JW. 2012. CysteinyltRNA deacylation can be uncoupled from protein synthesis. PLOS One 7: e33072. doi:10.1371/journal.pone.0033072

Deutscher MP, Marlor CW, Zaniewski R. 1985. RNase T is responsible for the end-turnover of tRNA in Escherichia coli. Proc Natl Acad Sci 82: 6427-6430. doi:10.1073/pnas.82.19.6427

Dittmar KA, Mobley EM, Radek AJ, Pan T. 2004. Exploring the regulation of tRNA distribution on the genomic scale. J Mol Biol 337: 31-47. doi:10.1016/j.jmb.2004.01.024

Dittmar KA, Goodenbour JM, Pan T. 2006. Tissue-specific differences in human transfer RNA expression. PLoS Genet 2: e221. doi:10 .1371/journal.pgen.0020221

Dong H, Nilsson L, Kurland CG. 1996. Co-variation of tRNA abundance and codon usage in Escherichia coli at different growth rates. J Mol Biol 260: 649-663. doi:10.1006/jmbi.1996.0428

Dougan DA, Micevski D, Truscott KN. 2012. The N-end rule pathway: from recognition by $\mathrm{N}$-recognins, to destruction by AAA + proteases. Biochim Biophys Acta 1823: 83-91. doi:10.1016/j.bbamcr .2011.07.002

Duh Y, Hsiao Y-Y, Li C-L, Huang JC, Yuan HS. 2015. Aromatic residues in RNase T stack with nucleobases to guide the sequence-specific recognition and cleavage of nucleic acids. Protein Sci 24: 19341941. doi:10.1002/pro.2800

Dupasquier M, Kim S, Halkidis K, Gamper H, Hou Y-M. 2008. tRNA integrity is a prerequisite for rapid CCA addition: implication for quality control. J Mol Biol 379: 579-588. doi:10.1016/j.jmb.2008 .04 .005

Ferro I, Ignatova Z. 2015. Quantifying the 'escapers' among RNA species. Biochem Soc Trans 43: 1215-1220. doi:10.1042/ BST20150158

Garg RP, Qian XL, Alemany LB, Moran S, Parry RJ. 2008. Investigations of valanimycin biosynthesis: elucidation of the role of seryltRNA. Proc Natl Acad Sci 105: 6543-6547. doi:10.1073/pnas .0708957105

Gogakos T, Brown M, Garzia A, Meyer C, Hafner M, Tuschl T. 2017. Characterizing expression and processing of precursor and mature human tRNAs by hydro-tRNAseq and PAR-CLIP. Cell Rep 20: 1463-1475. doi:10.1016/j.celrep.2017.07.029

Green R, Noller HF. 2002. Ribosomes and translation. Annu Rev Biochem 66: 679-716. doi:10.1146/annurev.biochem.66.1.679

Hentzen D, Mandel P, Garel J-P. 1972. Relation between aminoacyltRNA stability and the fixed amino acid. Biochim Biophys Acta 281: 228-232. doi:10.1016/0005-2787(72)90174-8

Kelly KO, Deutscher MP. 1992. Characterization of Escherichia coli RNase PH. J Biol Chem 267: 17153-8.

Kirchner S, Cai Z, Rauscher R, Kastelic N, Anding M, Czech A, Kleizen B, Ostedgaard LS, Braakman I, Sheppard DN, et al. 2017. Alteration of protein function by a silent polymorphism linked to tRNA abundance. PLoS Biol 15: e2000779. doi:10 .1371/journal.pbio.2000779

Kuhn C-D, Wilusz JE, Zheng Y, Beal PA, Joshua-Tor L. 2015. On-enzyme refolding permits small RNA and tRNA surveillance by the CCA-adding enzyme. Cell 160: 644-658. doi:10.1016/j.cell.2015 .01 .005

Lee CP, Mandal N, Dyson MR, RajBhandary UL. 1993. The discriminator base influences tRNA structure at the end of the acceptor stem and possibly its interaction with proteins. Proc Natl Acad Sci 90: 7149-7152. doi:10.1073/pnas.90.15.7149

Li Z, Deutscher MP. 1994. The role of individual exoribonucleases in processing at the $3^{\prime}$ end of Escherichia coli tRNA precursors. $J$ Biol Chem 269: 6064-6071.

Li Z, Deutscher MP. 2002. RNase E plays an essential role in the maturation of Escherichia colitRNA precursors. RNA 8: 97-109. doi:10 .1017/S1355838202014929

Linsen SE V, de Wit E, Janssens G, Heater S, Chapman L, Parkin RK, Fritz B, Wyman SK, de Bruijn E, Voest EE, et al. 2009. Limitations and possibilities of small RNA digital gene expression profiling. Nat Methods 6: 474-476. doi:10.1038/nmeth0709-474

Lizano E, Scheibe M, Rammelt C, Betat H, Mörl M. 2008. A comparative analysis of CCA-adding enzymes from human and E. coli: differences in CCA addition and TRNA $3^{\prime}$-end repair. Biochimie 90: 762-772. doi:10.1016/j.biochi.2007.12.007

Rak R, Dahan O, Pilpel Y. 2018. Repertoires of tRNAs: the couplers of genomics and proteomics. Annu Rev Cell Dev Biol 34: 239-264. doi:10.1146/annurev-cellbio-100617-062754

Reuven NB, Deutscher MP. 1993a. Multiple exoribonucleases are required for the $3^{\prime}$ processing of Escherichia coli tRNA precursors in vivo. FASEB J 7: 143-148. doi:10.1096/fasebj.7.1.8422961

Reuven NB, Deutscher MP. 1993b. Substitution of the $3^{\prime}$ terminal adenosine residue of transfer RNA in vivo. Proc Natl Acad Sci 90: 4350-4353. doi:10.1073/pnas.90.10.4350

Ruan B, Söll D. 2005. The bacterial YbaK protein is a Cys-tRNA ${ }^{\text {Pro }}$ and Cys-tRNA ${ }^{\text {Cys }}$ deacylase. J Biol Chem 280: 25887-25891. doi:10 $.1074 / j b c . M 502174200$

Shimizu Y, Inoue A, Tomari Y, Suzuki T, Yokogawa T, Nishikawa K, Ueda T. 2001. Cell-free translation reconstituted with purified components. Nat Biotechnol 19: 751-755. doi:10.1038/90802

Sørensen MA, Kurland CG, Pedersen S. 1989. Codon usage determines translation rate in Escherichia coli. J Mol Biol 207: 365 377. doi:10.1016/0022-2836(89)90260-X

van Dijk EL, Jaszczyszyn Y, Thermes C. 2014. Library preparation methods for next-generation sequencing: tone down the bias. Exp Cell Res 322: 12-20. doi:10.1016/j.yexcr.2014.01.008

Varshavsky A. 2011. The $\mathrm{N}$-end rule pathway and regulation by proteolysis. Protein Sci 20: 1298-1345. doi:10.1002/pro.666

Walker SC, Engelke DR. 2006. Ribonuclease P: the evolution of an ancient RNA enzyme. Crit Rev Biochem Mol Biol 41: 77-102. doi:10 $.1080 / 10409230600602634$

Wellner K, Czech A, Ignatova Z, Betat H, Mörl M. 2018. Examining tRNA 3 '-ends in Escherichia coli: teamwork between CCA-adding enzyme, RNase T, and RNase R. RNA 24: 361-370. doi:10.1261/ rna.064436.117

Wende S, Bonin S, Gotze O, Betat H, Morl M. 2015. The identity of the discriminator base has an impact on CCA addition. Nucleic Acids Res 43: 5617-5629. doi:10.1093/nar/gkv471

Wilusz JE. 2015. Removing roadblocks to deep sequencing of modified RNAs. Nat Methods 12: 821-822. doi:10.1038/nmeth .3516

Wilusz JE, Whipple JM, Phizicky EM, Sharp PA. 2011. tRNAs marked with CCACCA are targeted for degradation. Science 334: 817 821. doi:10.1126/science.1213671

Yakunin AF, Proudfoot M, Kuznetsova E, Savchenko A, Brown G, Arrowsmith $\mathrm{CH}$, Edwards AM. 2004. The HD domain of the 
Escherichia coli tRNA nucleotidyltransferase has $2^{\prime}, 3^{\prime}$-cyclic phosphodiesterase, 2'-nucleotidase, and phosphatase activities. J Biol Chem 279: 36819-36827. doi:10.1074/jbc.M405120200

Zaborske J, Pan T. 2010. Genome-wide analysis of aminoacylation (charging) levels of tRNA using microarrays. J Vis Exp doi:10 $.3791 / 2007$

Zaborske JM, Narasimhan J, Jiang L, Wek SA, Dittmar KA, Freimoser F, Pan T, Wek RC. 2009. Genome-wide analysis of tRNA charging and activation of the elF2 kinase Gcn2p. J Biol Chem 284: 25254-25267. doi:10.1074/jbc.M109.000877

Zhang G, Hubalewska M, Ignatova Z. 2009. Transient ribosomal attenuation coordinates protein synthesis and co-transla- tional folding. Nat Struct Mol Biol 16: 274-280. doi:10.1038/ nsmb.1554

Zheng G, Qin Y, Clark WC, Dai Q, Yi C, He C, Lambowitz AM, Pan T. 2015. Efficient and quantitative high-throughput tRNA sequencing. Nat Methods 12: 835-837. doi:10.1038/nmeth .3478

Zuo Y, Deutscher MP. 1999. The DNase activity of RNase T and its application to DNA cloning. Nucleic Acids Res 27: 4077-4082. doi:10.1093/nar/27.20.4077

Zuo Y, Deutscher MP. 2002. The physiological role of RNase T can be explained by its unusual substrate specificity. J Biol Chem 277: 29654-29661. doi:10.1074/jbc.M204252200 

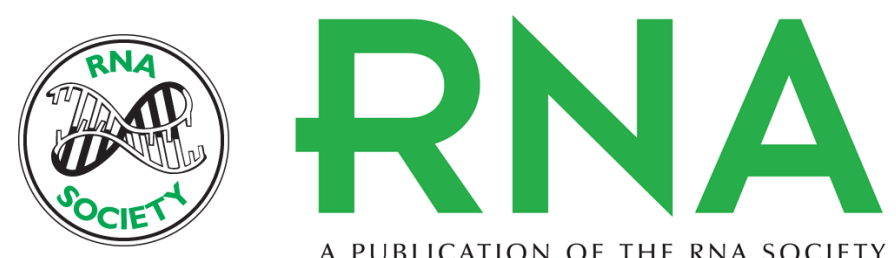

A PUBLICATION OF THE RNA SOCIETY

\section{Deep sequencing of tRNA's 3'-termini sheds light on CCA-tail integrity and maturation}

Andreas Czech

RNA 2020 26: 199-208 originally published online November 12, 2019

Access the most recent version at doi:10.1261/rna.072330.119

\section{Supplemental http://rnajournal.cshlp.org/content/suppl/2019/11/12/rna.072330.119.DC1 Material}

References This article cites 50 articles, 16 of which can be accessed free at: http://rnajournal.cshlp.org/content/26/2/199.full.html\#ref-list-1

Creative This article is distributed exclusively by the RNA Society for the first 12 months after the Commons License full-issue publication date (see http://rnajournal.cshlp.org/site/misc/terms.xhtml). After 12 months, it is available under a Creative Commons License (Attribution-NonCommercial 4.0 International), as described at http://creativecommons.org/licenses/by-nc/4.0/.

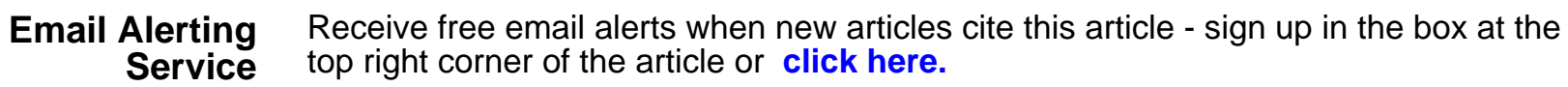

